\title{
La cultura come progetto educativo di una narrazione complessa
}

Culture as an educational project of a complex narrative

\author{
Dr. Salvatore Patera \\ Universidad del Salento \\ toto.patera@gmail.com
}

\section{Astratto}

La sfida educativa e culturale della complessità impone un pensiero aperto di tipo multilogico anziché monologico che considera una molteplicità di percorsi, punti di vista e di prospettive inclusive quale tensione culturale ed educativa alla complessità. L'educabilità dell'intersoggettività rappresenta la tensione dialogica, plurale e aperta per promuovere un processo interculturale di comprensione e di interdipendenza basato sulla reciprocità (autonomia-interdipendenza). L'intersoggettività, in quanto spazio relazionale e dialogico necessita una postura riflessiva. La narrazione, all'interno di un dispositivo educativo è in grado di potenziare la consapevolezza (e quindi competenze e capacità) nel leggere e agire la relazione con l'ambiente. La specificità educativa si situa quindi nel promuovere processi di apprendimento che tengono insieme riflessione e narrazione, scrittura e meta-cognizione lungo un processo di rilettura (e ri-scrittura) della propria storia e della propria cultura in quella più ampia della propria comunità e della società. La narrazione può ri-configurare la tensione tradizione-innovazione, conservazione-cambiamento.

Parole chiave: Complessità, Intersoggettività, Apprendimento, Riflessione, Narrazione

\begin{abstract}
The educational and cultural challenge of complexity requires an open mind to multilogical rather than monologic one considering a variety of routes, points of view and perspectives in terms of an inclusive cultural and educational tension to complexity. The educability of intersubjectivity is the dialogical, plural and open tension to promote an intercultural process of understanding and interdependence based on reciprocity (autonomy-interdependence). Intersubjectivity, as relational space and dialogue, needs a reflexive posture. The narrative, thought in an educational device, is able to enhance the
\end{abstract}


awareness (and thus skills and capacity) to read and act the relationship with the environment. The educational specificities which therefore fell in promoting learning processes that hold together reflection and storytelling, writing and meta-cognition along a proofreading and re-writing process of individual histories and cultures in the broadest of their community and society. The narrative can re-configure the traditioninnovation, conservation-change, tension.

Palabras clave: Complejidad, Intersubjetividad, Aprendizaje, Reflexión, Narración

Keywords: Complexity, Intersubjectivity, Learning, Reflection, Storytelling

\section{Complessità, partecipazione, apprendimento.}

La complessità emergente espressa da numerose questioni sociali, politiche, economiche, culturali ed educative nella contemporaneità, appare comprensibile all'interno della più ampia crisi globale che sta mandando in frantumi modelli decisionali e culturali auto-referenziali, tautologici, monologici quali visioni del mondo privilegiate. All'interno di tali modelli, e delle visioni culturali che li producono, l'effervescenza di diverse prospettive di cui sono portatori diversi attori, le istanze partecipative di numerosi punti di vista, la costruzione sociale di molteplici narrazioni di soggetti, gruppi, comunità e i bisogni che questi ultimi esprimono, sono sistematicamente esclusi dai processi di produzione di senso e da quelli decisionali (Crouch, 2003). Questi soggetti e le loro istanze sono considerati destinatari e spettatori di scelte effettuate da logiche di potere (culturale ed economico) dominanti (Sassen, 1992; Le Galès, 2002). Allo stesso tempo, tramite la partecipazione, nuovi attori esprimono una mappa complessa di bisogni, desideri, saperi locali, competenze che chiedono cittadinanza e che non sono riconosciuti dalle forme vacillanti di rappresentanza politica (Dahl, 1992; Beck, 2000). La sfida glocale, partecipativa e interculturale che richiede la complessità contemporanea, più che una nuova moda ideologica, rappresenta un progetto metodologico, e quindi pedagogico e sociale per superare le contraddizioni sottostanti a paradigmi culturali antagonisti, lungo il continuum locale-globale (Mander, Goldsmith, 2000). Ciò significa concentrare l'agire educativo e la riflessione pedagogica sull'interazione delle molteplici visioni nelle reciproche differenze lungo una costante tensione tra universalizzazione e localismo. In questa direzione l'universalizzazione rappresenta la sfida pedagogico-educativa che può darsi solo a partire di un dialogo interculturale con le "ontologie locali" all'interno di un percorso inclusivo, interculturale, dialogico che "coltivi l'intersoggettività" come pluralità di valori (Morin, 2000). La fine delle grandi narrazioni totalizzanti (Lyotard, 1981) che hanno polarizzato il dibattito culturale e politico dell'ultimo secolo, affini alla definizione dei miti d'oggi di Barthes (Barthes, 1974) e della doxa di Bourdieu (Bourdieu, 2003), si riferiscono alla crisi del "senso comune" e dell'ordine sociale e culturale cristallizzato verso il quale si era indirizzata, tra le altre, la critica di Gramsci (Gramsci, 1934). La crisi del senso comune e l'erosione alle egemonie culturali 
dominanti (Gruppi, 1972) ha a che fare con la convinzione storica maturata fino alla tardo-modernità che tali narrazioni fossero stabili e definitive piuttosto che costruzionicostrizioni culturali prodotte da conflitti di potere lungo la storia umana. Il carattere di incertezza della condizione contemporanea si situa proprio nella parzialità culturale e scientifica di visioni culturali concepite antagoniste e reciprocamente auto-sufficienti ed escludenti (aut-aut). Il termine crisi designa la rottura di un equilibrio precedentemente costituito e implica la trasformazione dei consueti schemi interpretativi e di azione che si rivelano inadeguati per i soggetti coinvolti rispetto alle nuove esigenze del contesto e dei contenuti della relazione. (Morin, 1980, p. 211). In tal senso, la crisi della modernità ha a che fare con la mutata percezione di non poter spiegare e agire la realtà all'interno di un approccio oppositivo e dialettico ove si presuma la presenza di:

- un punto di vista privilegiato ab interno quale riferimento su una antagonista visione culturale;

- $\quad$ un punto di vista privilegiato ab esterno, che superi tale dualismo.

La condizione postmoderna e la presenza di un "pensiero debole" esige di considerare la necessità di un dialogo interculturale tra differenti paradigmi e approcci lì dove la complessità abilita una molteplicità di punti di vista che non possono essere sintetizzati in maniera esclusiva ed escludente attraverso l'adozione di un "metapunto di vista" (Morin, 1974). La nostra epoca, non ancora fuoriuscita dalla modernità (tarda modernità) è caratterizzata da una dimensione riflessiva di auto-critica (Beck, Giddens, Lash, 1999). Il declino della modernità e il suo superamento passa dalla possibilità dialogica e plurale di trovare risposte complesse, plurali e includenti e un linguaggio condiviso in grado di far fronte alla complessità nei continui equilibri che tali momenti di incertezze provocano tra paradigmi culturali antitetici (istanze istituite e istituenti).

Questo scontro paradigmatico e culturale rinvenibile nell'approccio tradizionale dialettico pone oggigiorno la necessità dialogica della co-esistenza di prospettive che lavorano nella direzione del cambiamento sociale focalizzandosi sulle interconnessioni tra il livello bottom up e quello top-down. Infatti, rispetto allo stallo e alla radicalizzazione del dibattito tra paradigmi culturali contrapposti e antagonisti si è via via generata una situazione di doppio vincolo, che rischia di produrre su scala nazionale e internazionale una schismognesi. (Watzlawick, et al, 1967, pp. 99-111). Tale paralisi comunicativa pone in maniera ambivalente processi di disintegrazione del sistema istituito di fronte all'irruzione di forze istituenti oppure di rafforzamento del sistema istituito a fronte di un annientamento (dissolvimento-fagocitazione) delle specificità e delle differenze di cui sono portatrici le molteplici istanze emergenti. Infatti, il doppio vincolo, incapace di pensare in termini includenti (dialogici piuttosto che dialettici) tende a generare il doppio vincolo. Questo doppio vincolo comunicativo trova il suo superamento seguendo un'opzione dialogica e transculturale (vel-vel) basata sul riconoscimento reciproco a fronte di un processo dinamico di mutua identificazione culturale (Geertz, 1998). Nel superamento di questo vincolo dialettico tra differenti posizioni culturali è possibile riconfigurare il rapporto di coesistenza tra i molteplici livelli lungo un processo di mutuo riconoscimento, di co-costruzione sociale di senso (Morin, 1995, p. 43). La sfida educativa e culturale della complessità impone l'adozione di un pensiero aperto di tipo multi-logico anziché monologico che considera una molteplicità di percorsi, punti di vista e di prospettive inclusive quale tensione culturale 
ed educativa alla complessità. In tal senso, lo stesso Visalberghi considera la necessità di promuovere un'educazione al pensiero complesso ove, l'azione educativa e la riflessione pedagogica possano educare ad una modalità di pensiero aperta, flessibile, e dunque complessa (Visalbegrhi, 1987). In questo senso, tale processo deve basarsi sulla comprensione, quale progetto di convivenza sociale da co-costruire all'interno di un'etica e di una cultura plurale, dialogica, aperta, planetaria (Morin, 2001). Infatti, come sostengono Maturana e Varela: "Crescere come membro di una società consiste nel diventare strutturalmente accoppiato ad essa;” (Maturana, Varela, 1985, p. 42).

Ossia, una tensione, direbbe Bateson a partire dalla necessità di credere sia nell'azione collettiva che nell'inevitabile tensione con cui il desiderio individuale le si oppone (Bateson, 1976). I sistemi complessi sono auto-eco/organizzati, infatti, nella cornice ecologico-sistemica esiste una "via di mezzo" fra autonomia ed eteronomia che permette di pensare "la co-emergenza delle unità autonome e dei loro mondi" (Varela, 1985, p. 132). Da questo punto di vista un sistema è per definizione, auto-poietico ossia possiede la capacità di auto-costruirsi, rimanendo aperto alle influenze e perturbazioni dell'ambiente esterno (Maturana, H., Varela, 1987). Questa continua ri-definizione del sistema verso un equilibrio dinamico rappresenta la condizione dell'autonomia degli agenti nell'interazione sociale, la quale, sarebbe dovuta proprio alla loro "eteronomia" nel senso che le "costrizioni"normative esterne possono essere configurate come "risorse" (capacità e competenze) che rendono possibile agire in relazione con gli altri (Steiner, Stewart, 2009, p. 527). La crisi percepita come opportunità, può rappresentare il punto di avvio di una relazione comunicativa di tipo ecologico-sistemico quale tensione dinamica verso la co-evoluzione tra differenti punti di vista lungo un processo di deutero-apprendimento in grado di accrescere la capacità dei sistemi sociali e culturali di auto-co/organizzarsi migliorando la propria autonomia nell'interdipendenza con altri sistemi lungo una reciprocità di scambi comunicativi (Zizek, 2012).

\section{Agire pedagogico come progetto comunicativo dialogico e interculturale}

Rispetto al discorso intrapreso, ci torna utile il costrutto di cultura intesa come "ragnatela di significati" formulato, in maniera, autorevole da Geertz: "Una struttura di significati trasmessa storicamente, incarnati in simboli, un sistema di concezioni ereditate espresse in forme simboliche per mezzo di cui gli uomini comunicano, perpetuano e sviluppano la loro conoscenza e i loro atteggiamenti verso la vita" (Geertz, 1987, p. 141). In linea con quanto esplicitato nel paragrafo precedente, Maturana e Varela la definiscono "reciprocità di comportamenti significativi" (Maturana, Varela, 1987). L'ordine simbolico dato dalla coesistenza in un ambiente comune (il mondo della vita di Husserl) si co-costruisce attraverso pratiche sociali e relazioni che hanno alla base, come sosteneva anche Mead, e poi Vygotskij e Habermas, il linguaggio (Mead, 1966). Berger e Luckmann considerano il senso comune "la conoscenza che io condivido con altri nelle normali, auto-evidenti routine della vita quotidiana" (Berger, Luckmann, 1979, p. 44). Il senso comune, come corso di azione ritualizzato, è il sistema di regole (non solo comunicative) a partire dalle quali si forma la cultura dominante. In tal senso le guerre, sosteneva Wallerstein, sono guerrre di significati, ossia, principalmente di tipo semantico. (Wallerstein, 1974). Ma è il mondo stesso che nell'interpretazione postmoderna emerge come complessa interrelazione di "processi locali in continua lotta e negoziazione su significati, valori e risorse. Siamo tutti 
architetti della "modernità" e "cannibali dell'ordine sociale" (Bauman, 1999). Ciò appare coerente con quanto definito da Habermas, il quale, ha affrontato la cultura rispetto al nesso che la lega fortemente al binomio comunicazione e potere Habermas (1996). Nella contemporaneità, appare comunque vero il fatto che, tali regole sono decise in gran parte dalla relazione di potere con il sistema dominante e sono basate proprio sulla cristalizzazione del senso comune (attraverso una comunicazione ridondante e ricorsiva). "I confini e le gerarchie sono sempre stabiliti da un osservatore, le cui operazioni e le cui decisioni intervengono a più livelli nel processo di costruzione di un sistema" (Bocchi, Ceruti, 2007, p.8). Tale senso comune all'interno di una comunità parlante è il terreno educativo del cambiamento culturale e sociale su quale Gramsci poneva l'accento a partire dalla promozione del senso critico. Il senso critico è un processo di riflessione sulla propria esperienza, una narrazione in termini di riconfigurazione di senso (deutero-apprendimento) in grado di rileggere e agire i nessi tra vissuti individuali e collettivi (Bateson, 1976). Per Habermas (1996), l'agire comunicativo deve essere pensato come agire interculturale ove l'etica della comunicazione consideri l'intersoggettività quale presupposto per costruire una comunità della comunicazione. In ogni modo il "senso di realtà" cui Bauman rimanda, quale atto di responsabilità verso l'alterità e l'autonomia nel reciproco riconoscimento dell'altro, considera l'importanza della società quale senso etico e civile. Come specifica Bauman 'assumersi la responsabilità verso l'alterità, l'identità e l'autonomia dell'altro è una condizione cruciale per la realizzazione di ogni individuo ed e' una parte indispensabile di ogni autentica "pragmatica dello scambio" (Bauman, 1999).

L'esistenza si manifesta come un continuo tendere verso l'apertura intersoggettiva, esperendo la presenza dell'alterità nel divenire umano. La comunicazione non di tipo dialettico, tendente alla schismogenesi tra istanze centrifughe-centripete di eteronomiaautonomia, bensì di tipo ecologico-sistemica, rappresenta un progetto educativo per pervenire a un set di regole concordate in una "comunità parlante": un progetto interculturale e dialogico chesi basa su una sfida educativa a promuvoere capacità e competenze che vadano in questa direzione (Le Boterf, 2008). L'esistenza quindi ha a che fare con una tensione all'autonomia che si esplica in una dimensione dialogica, interculturale, plurale ossia in una condizione relazionale di intersoggettività: autorappresentandosi come intenzionalità che si apre ad altri soggetti. L'intersoggettività quale processo educabile secondo un approccio ecologico-sistemico, può assumere una funzione mediatrice in quanto genera "scambi simbolici" di tipo comunicativo tra $i$ soggetti ch prendono paete a un processo comunicativo. Tali scambi simbolici definiscono la mediazione del mondo che si realizza tramite il linguaggio. L'educabilità dell'intersoggettività come costrutto pedagogico rappresenta la tensione dialogica, plurale e aperta per promuovere un processo interculturale di comprensione e di interdipendenza basato sulla reciprocità. La reciprocità è da intendere quindi come tensione dialogica basata sugli aspetti di significato in comune sul quale co-costruire un progetto di convivenza culturale di "cittadinanza planetaria" in termini di reciprocità di comportamenti significativi basati su consenso, intersezione, ragionevolezza. Nel mondo che viviamo in comune con gli altri (Lebenswelt) l'intersoggettività, in quanto spazio relazionale e dialogico è caratterizzata da una postura riflessiva e di osservazione interna (cibernetica di secondo ordine) che diviene autocoscienza. Tale intersoggettività viene definita da Morin autoriflessione universale. Per Freire e la pedagogia libertaria, a partire da un processo di auto-consapevolezza della propria condizione, si realizza la 
tensione verso l'altro, come possibilità di dialogo. "Il dialogo è questo incontro di uomini, attraverso la mediazione del mondo, per dargli un nome, e quindi non si esaurisce nel rapporto io-tu"(Freire, 2002, p. 78). L'intersoggettività come coinvolgimento cognitivo ed emotivo ha a che fare quindi con "L'esserci in quanto essere-nel mondo è contemporaneamente un essere-l'uno-con-l'altro, un essere con altri” (Heidegger, 2012, p. 32). A tal proposito Ricoeur nell'opera Sé come un altro pone la questione dell'identità nella misura in cui l'alterità è implicata a un livello originario e profondo nel processo di costituzione del sé (Ricoeur, 1993). La pedagogia lavora con l'intersoggettività ed è interessata alla costruzione di un senso critico e allo sviluppo di competenze e capacità per migliorare l'adattamento reciproco tra persona e ambiente (es soggetti, gruppi, comunità, istituzioni) attraverso un processo di negoziazione tra le diverse prospettive e di co-costruzione sociale finalizzato proprio all'equilibrio dinamico di tipo auto/co-evolutivo. Secondo la prospettiva sistemicocostruttivista i soggetti attribuiscono senso e valore alla propria esperienza interagendo con gli altri a partire dal complesso di personali beliefs (credenze, valori, atteggiamenti, ecc) le quali derivano dal contesto e dalla collocazione all'interno dell'interazione e contribuiscono tramite un processo di re-interpretazione, alla ri-definizione del contesto stesso e delle sue regole(Garfinkel, 1974, pp. 15-18). Nel processo comunicativo, le rappresentazioni del soggetto, mediate dal contesto culturale cui prende parte (senso comune) e quindi dalle rappresentazioni degli altri, consentono, tramite continui feedback (retroazioni positive/negative) di dare significato all'agire sociale allo scopo di mantenere una coerenza all'interno del proprio sistema di rappresentazioni e fra questo e il proprio comportamento rispetto agli altri (Peirce, 1994). La comunicazione permette non solo lo scambio di informazioni da codificare-ricodificare a partire da differenti sistemi di premesse (beliefs) ma anche la negoziazione di significati a partire da tali punti di vista differenti. Tale processo di negoziazione di significati è un processo educativo di co-costruzione sociale che contribuisce a definire al contempo le identità individuali e le identità collettive. Il carattere di embricazione tra il livello individuale e quello sociale ossia il dialogo tra sistemi comunicativi è definito da Bateson come "the patterns which connect"(Bateson, 1972) e avviene su due livelli: la costruzione individuale e la co-costruzione collettiva. In tal senso, la dimensione individuale, comunque embricata in quella sociale, modula istanze di continuità-cambiamento per permettere la relazione con gli altri ossia rinvenendo strategie atte a mantenere una coerenza tra premesse e comportamenti a partire dai significati attribuiti ai comportamenti di sé e degli altri (Goffmann, 1988). Il secondo livello è riferito al processo di co-costruzione della realtà quale processo sociale a partire dalle interazioni comunicative attivate da differenti sistemi sociali intesi come attori della comunicazione. Tale secondo livello ha a che fare con i processi di negoziazione di beliefs (significati, valori, ecc) che gli attori realizzano attraverso scambi linguistici per sintonizzarsi con gli altri compartecipando alla co-costruzione della realtà. In quest'ottica, la dimensione educativa è finalizzata a promuvoere processi di cambiamento quale attività situata ed emergente dall'interazione tra un attore cognitivo e i vincoli contestuali, attività, distribuita e condivisa, cui concorrono la natura e la storia delle relazioni, tra gli individui coinvolti, le caratteristiche fisiche dell'ambiente, gli strumenti improntati dalla cultura (Liverta Sempio, Marchetti, 1995). Promuovere la riflessività degli attori e dei loro 'mondi' equivale a stimolare la loro capacità di (auto)progettarsi di fronte ai paradossi e alle ambiguità dei fenomeni di svuotamento della tradizione, di messa in dubbio delle certezze razionali e della stabilità delle 
conoscenze. Dewey infatti, afferma che "imparare a essere umani significa sviluppare quel fare e ricevere in cui consiste la comunicazione" (Dewey, 1971, p. 121). In tal senso, "la pedagogia, che lavora sì per rendere adattato il soggetto al suo ambiente, ma soprattutto per renderlo capace di progettualità, mediante la quale lavorare per adattare il suo ambiente a sé, ai suoi bisogni, ai suoi desideri, alla sua immaginazione" (Colazzo, 2011, p. 8). Educare al cambiamento sociale significa pertanto monitorare e facilitare interventi educativi volti a creare occasioni e stimoli per riflettere sul funzionamento della società e sui vincoli che essa impone rinvenendo strategie di autonomia e di azione che sappiano tendere tra le istanze di trasmissione sociale e di trasformazione sociale.

La pedagogia come regolatore comunicativo e per il suo carattere di mediatività, tende all'obiettivo di realizzare l'uomo nella società tendendo all'equilibrio dinamico di convivenza tra le differenze ossia considerando cambiamento e stabilità come processi complementari. "La pedagogia della postmodernità può lavorare per essere coeva al suo tempo, a costruire la libertà e l'autonomia dei soggetti, favorendo la loro attività di decisione e di scelta, promuovendo il costituirsi di una forma di razionalità aperta all'intersoggettività, al dialogo, alla discussione, a gusto della negoziazione di significati" (Colazzo 2005, p. 29). In tal senso il processo di co-costruzione della conoscenza e della cultura si definisce per il suo carattere intrinsecamente intersoggettivo e di auto/co-costruzione sociale a partire dal contesto quotidiano nel quale si consumano retroazioni positive e retroazioni negative alla ricerca di continui equilibri in rapporto tra differenti sistemi sociali e molteplici riferimenti culturali. A proposito dei costrutti di "senso comune gramsciano", della "Doxa" di Bourdieu, delle "false credenze di peirce" la letteratura sistemico-costruttivista ci ha informati sul fatto che i processi di riflessione, deutero-apprendimento, metacognizione rappresentano la sfida educativa per rileggere se stessi e la relazione tra se stessi e il contesto culturale nel quale si vive. In tal senso, l'apprendimento non fa che promuovere processi di rilettura dei limiti individuali e contestuali individuando strategie e risorse per agire la complessità contemporanea. Archer (2006), sottolinea l'influenza della cultura sulle persone e la capacità di questi ultimi di attingere alla "conversazione interiore" proprio per comprendere e agire il sociale. Tale conversazione interiore rappresenta una modalità riflessiva per rielaborare quanto emerge dall'esterno ossia dalla struttura sociale. Il dialogo tra inconscio/conscio - cognitivo e inconscio/conscio sociale rappresenta la possibilità di adattarsi creativamente alla società avendo perlopiù margini di libertà e di trasformazione partendo proprio dalla rielaborazione $a b$ interno delle sollecitazioni e dei vincoli espressi dalla società e dalla cultura ponendo in connessione la dimensione tacita e inconscia delle preferenze lavora proprio sul potenziamento di tali competenze e capacità. L'auto-riflessività permette di collegare il mondo interno con il mondo esterno attraverso un approccio ermeneutico e basato sul costruzionismo sociale che permette di connettere la trama dei vissuti individuali a quelli collettivi. In questo modo la narrazione biografica permette di riconfigurare l'identità come un processo di co-costruzione sociale. In tal modo, la narrazione delle persone, dei gruppi, delle comunità scrive e si inscrive in una narrazione più ampia che è la memoria collettiva (Levy, 1996) e connettiva di tipo sociale (De Kerckhove, 1997).

Recentemente un testo importante come quello curato da Kaneklin e Scaratti (1998) ha ribadito il valore della narrazione come strumento indispensabile sia per la costruzione di significati, di pratiche simboliche e di artefatti materiali e immateriali (cognitivo- 
culturali) sia per la facilitazione dei processi di cambiamento sociale ed organizzativo in contesti sociali ed educativi. Il punto di vista narrativo risulta infatti connesso alla modalità esperite dai soggetti di attribuzione di senso agli eventi e alla realtà di comunicazione come agire sociale. L'educazione è quindi intesa non solo come trasmissione di conoscenze ma come relazione di persone che si scambiano esperienze attraverso la narrazione e l'ascolto reciproco (Mantegazza, 1999).

\section{Riflessione, narrazione, memoria connettiva}

Problematizzare la nostra cultura, non per distruggerla, ma per farne scaturire le possibilità taciute nei secoli attraverso un processo maieutico" (Freire, 1971, p. 11).

Come abbiamo sintetizzato nei paragrafi precedenti, l'homo narrans rappresenta una condizione storica rinvenibile a partire dalla preistoria e rappresenta un'esigenza relazionale di profonda rilettura della propria esperienza di vita quotidiana all'interno di contesti culturali più ampi (Benjamin, 1976, p. 235), (Eco, 1993). Ciò appare ancora più cruciale nella complessità contemporanea, per via dell'assenza dei Miti unitari e monolitici a cui facevano riferimento, tra gli altri, Barthes e Lyotard con rispetto alla cultura intesa in maniera organica, gerarchica. Ossia, l'impossibilità di dare una rappresentazione esaustiva, totalizzante e tranquillizzante della realtà, che possa "ridurre" la complessità nella quale ci troviamo a vivere e che contempli la cultura come narrazione condivisa e statica. Hall, a tal proposito osserva come "le identità sono relative alle modalità cui siamo posizionati e ci posizioniamo nelle numerose narrazioni" (Hall, 1990, p. 222). Il costrutto di deutero-apprendimento, come abbiamo evidenziato nelle pagine precedenti permette di rileggere la propria biografia, memoria, narrazione in quanto interdipendente con il contesto comunitario, sociale che modifichiamo e dal quale siamo modificati lungo una tensione tra simmetria e complementarietà (autonomia-eteronomia). In questo modo la narrazione biografica permette di riconfigurare l'identità come un processo di co-costruzione sociale in cui la narrazione delle persone, dei gruppi, delle comunità scrive e si inscrive in una narrazione più ampia che è la memoria collettiva e connettiva di tipo sociale. L'utilità di adottare un dispositivo educativo basato sul nesso memoria-narrazione permette di rileggere il vissuto dei soggetti e delle comunità per rinvenire margini di ri-definizione del proprio senso nel sociale e del proprio orientamento nella società. Come sostiene $M$. Callari Galli, il passato culturale e la memoria delle comunità non va considerato solo come uno strumento per promuovere la partecipazione di un soggetto alla propria comunità bensì diventa uno slancio verso un processo di co-costruzione quale nuova progettualità culturale. In tal modo è possibile ri-configurare pezzi della memoria del passato per connettere le generazioni future ad un progetto di partecipazione e nuova cittadinanza alla vita della comunità (Callari Galli, et al, 1998, pp. 191-192). Tale opportunità di uscire dal vincolo comunicativo posto da una discorso, cultura, memoria dominante ha a che fare con la possibilità di ri-configurare, all'interno di un processo educativo, un senso condiviso interculturale e intersoggettivo dei soggetti nelle comunità e nella più ampia società quale tensione in divenire e spazio educativo di costruzione socio-culturale della convivenza civile. La narrazione individuale e di gruppo, quale processo riflessivo di deutero-apprendimento può ri-configurare la tensione tradizione-innovazione, conservazione-cambiamento. Riprendendo il contributo di Bateson sul deutero-apprendimento, il processo di ri-memorazione 
permette una definizione delle proprie coordinate esistenziali nel presente-passatofuturo attraverso una crisi propositiva per ri-configurare equilibri complessi tra istanze di conservazione-mutamento tra persona-comunità società (individuo-ambiente). Al riguardo, in uno dei suoi lavori più significativi, Giddens afferma che le strutture sociali sono di fatto tracce della memoria rinvenibili nelle pratiche quotidiane (Giddens, 1990).

La memoria collettiva e connettiva della comunità rappresenta una narrazione mediata e socialmente costruita nelle relazioni e nelle interazioni con le memorie biografiche dei soggetti. Oltre una prospettiva funzionalista di memoria collettiva proposta da Halbawchs che tende a sottolinearne il carattere proprio di staticità e di continuità, in questa prospettiva essa assume una connotazione abduttiva, fatta cioè, di continue ridefinizioni tra memorie individuali emergenti e cristallizzazioni delle memoria istituita (senso comune). Narrare il sé è un modo per rileggersi e per rileggere se stessi tra vincoli e possibilità di trovare una strada praticabile all'autonomia nell'interdipendenza con il contesto culturale e sociale più ampio. Narrare è quindi agire poichè, in questi termini, il narrare mai descrive, agisce; su me che racconto, sull'altro che ascolta (Geertz, 1987), Jedlowski, ripercorrendo i nodi epistemologici della sociologia della vita quotidiana si concentra sull'importanza della vita quotidiana come spazio di riflessione, di narrazione e di ri-costruzione sociale della propria identità all'interno di un gruppo, comunità, società. Per promuovere il cambiamento della società è necessario partire dagli artefatti culturali che producono il senso comune ovvero, ciò che è più prossimo: la memoria collettiva. Ossia una coscienza collettiva delle interdipendenze tra le memorie biografiche individuali e quella connettiva delle comunità e della società che, più che essere un ancoraggio nostalgico al passato diviene uno slancio propositivo per proiettarsi in orizzonti di senso condivisi. La condizione di attraversamento come caratteristica dialogica delle identità contemporanee è espressa anche in ambito antropologico da autori come Clifford e Geertz. Entrambi pongono la questione antropologica contemporanea come postcoloniale a partire dal considerare la cultura translocale che esprime identità intese come "travelling cultures" che riflettono sulle proprie pratiche di attribuzione di senso "writing cultures" (Clifford, 1999, p. 19). Tali punti di vista, a seguito di continui aggiustamenti tra istanze di conservazionecambiamento, tradizione-innovazione ri-configurano nuovi equilibri più complessi.

Questo processo di negoziazione tra memorie, rappresentazioni, vissuti individuali e memorie collettive si costituisce come tensione proattiva al cambiamento che trova nel contributo teorico e metodologico della narrazione come dispositivo pedagogico una modalità efficace per monitorare e facilitare pratiche interculturali tra persone-gruppicomunità-società. Allo stesso modo il metodo narrativo favorisce un processo educativo di auto-riflessione, di auto-consapevolezza e di auto-progettualità nella tensione tra conservazione-cambiamento, tradizione-innovazione, istanze $\mathrm{e}$ rappresentazioni individuali e collettive. La narrazione rappresenta pertanto un processo di ri-configurazione identitaria della propria storia personale come prodotto e produttore di una storia comunitaria e sociale. La riflessione, attiva un processo riflessivo di rilettura dell'esperienza dell'esperienza (esperienza di secondo livello) proprio dell'approccio sistemico-costruttivista. Attraverso la narrazione, in quanto riflessione sull'esperienza, infatti, rileggiamo noi stessi e riscriviamo il senso del nostro agire quotidiano (Jedlowski, 2000). In tal senso c'è differenza tra il fare esperienza e l'avere esperienza. In questo secodo caso è insito nel riflettere il raccontarsi ossia la possibilità 
di non limitarsi al fatto in sé ma di rivestirlo di un altro significato accessibile ad altri mediante l'utilizzo della scrittura (Jedlowski, 1986). Infatti l'uomo odierno ha sete di narrazione perché nella narrazione ritrova spazio e tempo per la propria vita (Knowles, 1996). Dal punto di vista della valenza educativa della narrazione, come sostiene Bruner (1993), Il pensiero narrativo si occupa del particolare, delle intenzioni e delle azioni dell'uomo, delle vicissitudini e dei risultati. Il suo intento è quello di situare l'esperienza nel tempo e nello spazio.

Il lavoro educativo intende quindi promuovere lo sviluppo dell'intelligenza narrativa in quanto intelligenza ermeneutica, quale capacità che va alla ricerca del significato e degli altri, conoscenza di se stessi in relazione ad altri (Gardner, 2002). "Il mondo della vita quotidiana è la scena ed è anche l'oggetto delle nostre azioni ed interazioni. Dobbiamo dominarlo e cambiarlo per realizzare $i$ fini che perseguiamo in esso tra i nostri simili. (...) Il mondo in questo senso è qualcosa che dobbiamo modificare mediante le nostre azioni e che modifica le nostre azioni" (Schutz, 1975, p.13). La specificità educativa si situa quindi nel promuovere processi di apprendimento che tengono insieme riflessione e narrazione, scrittura e meta-cognizione lungo un processo di rilettura (e ri-scrittura) della propria storia e della propria cultura in quella più ampia della propria comunità e della società (Jedlowski, 1994) . In tal senso la memoria stessa in quanto processo selettivo rappresenta il perno su cui si costruisce l'identità tra passato, presente e futuro in una mediazione tra memoria individuale e memoria collettiva ossia tra istanze di trasmissione/riproduzione e istanze di re-interpretazione e ri-definizione della realtà. Questa mappatura delle forme di narrazione ci informa del fatto che il discorso si muove nella dinamica io-altro e non può mai sfuggire alla sua natura relazionale. L'identità quotidiana che è tale in quanto in relazione lungo un coinvolgimento in pratiche, si percepisce come soggetto, come persona che nella-della-sulla comunità si racconta. Outsider inside, propone Collins, oltre le false alternative paradossali del doppio vincolo svelate da Watzlawick (Collins, 1986). Lo scopo di questa relazione è di incidere, riportando segno tangibile di questo confine negoziato e praticato con gli altri, nella definizione di una narrazione connettiva. In tal senso la conoscenza non è un sistema gerarchico ma un sistema di co-costruzione e di interconnessioni che garantisce la continua evoluzione e ri-configurazione del sapere. La narrazione è in grado di potenziare una miglior consapevolezza della relazione con l'ambiente, nella singolarità della propria appartenenza inserendo la propria biografia in un universo di senso più ampio. La narrazione come strumento di consapevolezza delle memorie e delle biografie individuali permette la rilettura delle esperienze, delle tradizioni, delle biografie, dei racconti popolari, delle conoscenze tacite, dei saperi locali, nella consapevolezza che l'identità è un processo circolare emersivo/immersivo di negoziazione e co-costruzione sociale tra le diverse persone che vivono in una comunità. E' quindi un processo lento di stratificazioni, sincretismi ed equilibri contemporanei tra tradizione-innovazione, continuità-mutamento. Como sostiene Colom, la narrazione, sia in quanto processo individuale che collettivo, permette che la nostra percezione diventi partecipata e condivisa con gli altri traducendosi in artefatti simbolici e in pratiche divenendo in questo modo una metafora della stessa realtà, una costruzione sociale della realtà (Colom, 2002). 


\section{Referencias Bibliográficas}

Archer, M. S. (2006). La conversazione interiore. Come nasce l'agire sociale. Torino: Centro Studi Erickson.

Barthes, R. (1974). I miti d'oggi. Torino: Einaudi.

Bateson, G. (1972). Mente e natura. Milano: Adelphi.

Bateson, G. (1979). Verso una ecologia della mente. Milano: Adelphi.

Bauman, Z. (1999). La società dell'incertezza. Bologna: Il Mulino.

Beck, U., IN Beck, U., Giddens, A., Lash, S. (1999). Modernizzazione riflessiva. Trieste: Asterios.

Beck, U. (2000). La società del rischio. Roma: Carocci.

Benjamin, W. (1976). Considerazioni sull'opera di Nicola Leskov, in Angelus Novus. Torino: Einaudi.

Berger, P., Luckmann, T. (1969). La realtà come costruzione sociale. Bologna: Il Mulino.

Bocchi, G., Ceruti, M. (2007). La sfida della complessità. Milano: Feltrinelli.

Bourdieu, P. (2003). Per una teoria della pratica con tre studi di etnologia cabila. Milano: Ed. Cortina.

Bruner, J. (1993). La mente a più dimensioni. Bari-Roma: Laterza.

Callari Galli, M., Pievani, T., Ceruti, M. (1998). Pensare la diversità. Per un'educazione alla complessità umana. Roma: Meltemi.

Clifford, J. (1999). Strade. Viaggio e traduzione alla fine del secolo XX. Torino: Bollati Boringhieri.

Colazzo, S. (2011). La pedagogia alla luce del paradigma ecologicosistemico. Relazione tenuta al Convegno organizzato dalla SIPNEI (Società Italiana di Psiconeuroendocrinoimmunologia), Roma.

Colazzo, S. (2005). Insegnare ed apprendere in rete. Castrignano dei Greci: Amaltea.

Collins, P. H. (1986). Learning from the outsider within. Social Problems, n. $33,514-532$.

Colom, J. A. (2002). La (de)construcción del conocimiento pedagógico: nuevas perspectivas en teoría de la educación. Barcelona: Paidós.

Crouch, C. (2003). Postdemocrazia. Bari-Roma: Laterza.

Dahl, R. (1992). La democracia y sus críticos. Barcelona: Paidós.

De Kerckhove, D. (1997). Intelligenza connettiva. Avvento della web society. Roma: De Laurentis.

Dewey, J. (1971). Comunità e potere. Firenze: La Nuova Italia.

Eco, U. (1993). Sei passeggiate nei boschi narrativi. Milano: Bompiani.

Freire, P. (2002). La pedagogia degli oppressi, Torino: EGA.

Gardner, H. (2002). Formae mentis. Saggio sulla pluralita` dell'intelligenza. Milano: Feltrinelli.

Garfinkel, H. (1974). The origin of the term ethnomethodology, in Ethnomethodology (eds) Turner, R. Harmondsworth.

Geertz, C. (1987). Interpretazione di culture. Bologna: Il Mulino.

Giddens, A. (1990). La costituzione della società. Milano: Edizioni Comunità.

Goffman, E. (1988). Il rituale dell'interazione. Bologna: Il Mulino. 
Gramsci, A. (2007). Quaderni del carcere, (eds) Garretana, V. Torino: Einaudi.

Gruppi, L. (1972). Il concetto di egemonia in Gramsci. Roma: Editori Riuniti.

Habermas, J. (1996). Between Facts and Norms: Contributions to a Discourse Theory of Law and Democracy. Cambridge MA: MIT Press.

Hall, S. (1990). Cultural identity and Diaspora, in Rutherford, J., Identity: Community, Culture and Difference. London: Lawrence and Wishart.

Heidegger, M. (2010). Il concetto di tempo. Milano: Adelphi Edizioni.

Jedlowski, P. (2003). I fogli nella valigia. Sociologia e cultura. Bologna: Il Mulino.

Jedlowski, P. (1994). Il sapere dell'esperienza. Milano: Il Saggiatore.

Jedlowski, P. (1986). Il tempo dell'esperienza. Milano: Franco Angeli.

Jedlowski, P. (2000). Storie comuni. La narrazione nella vita quotidiana. Milano: Bruno Mondadori.

Kaneklin, C. Scaratti, G. (eds). (1998). Formazione e narrazione. Milano: Cortina.

Knowles, S. (1996). La formazione degli adulti come autobiografia. Milano: Cortina.

Le Boterf, G. (2008). Costruire le competenze individuali e collettive. Napoli: Guida.

Le Galès, P. (1992). European Cities: Social Conflicts and Governance. Cornwall: Oxford University Press.

Levy, P. (1996). L'intelligenza collettiva. Per un'antropologia del cyberspazio. Milano: Feltrinelli.

Liverta Sempio, O., Marchetti, A. (eds.) (1995). Il pensiero dell'altro. Contesto, conoscenza e teorie della mente. Milano: Raffaello Cortina.

Lyotard, J. F. (1981). La condizione postmoderna. Milano: Feltrinelli.

Mander, J., Goldsmith, E. (2000). Glocalismo, L'alternativa strategica alla globalizzazione. Bologna: Arianna.

Mantegazza, R. (1999). Un Tempo per narrare. Esperienze di narrazione a scuola e fuori. Torino: EMI.

Maturana, H. R.,Varela, F. J. (1987). L'albero della conoscenza. Milano: Garzanti.

Maturana, H., Varela, F. J. (1985). Autopoiesi e cognizione. Venezia: Marsilio.

Mead, G. H. (1966). Mente, sé e società. Firenze: Giunti e Barbera.

Morin, E. (2001). I sette saperi necessari all'educazione del futuro. Milano: R. Cortina.

Morin, E. (1974). Il paradigma perduto. Milano: Bompiani.

Morin, E. (1995). Introduzione al pensiero complesso. Milano: Sperling \& Kupfer.

Morin, E. (2000). La testa ben fatta. Riforma dell'insegnamento e riforma del pensiero. Milano: Cortina. 
Morin, E. (1980). Per una teoria della crisi, in D’Eramo, M., (eds). (1980). La crisi del concetto di crisi. Roma: Lerici.

Peirce, B. (1994). Interpersonal communication: making social worlds. New York: Harper Collins.

Ricoeur, P. (1993). Sé come un altro. Milano: JacaBook.

Sassen, S. (1992). The Global City, Princeton. New York: Princeton University Press.

Schutz, A. (1975). Il problema della rilevanza, Riconda, G., (eds). Torino: Rosenberg and Sellier.

Steiner, P., Stewart, J. (2009). From autonomy to heteronomy (and back): the enaction of social life, Phenomenology and the Cognitive Science, 8, 527-550.

Varela, F. (1985). Complessità del cervello e autonomia del vivente, in: Bocchi, G., Ceruti, M. (eds). La sfida della complessità. Milano: Feltrinelli.

Visalberghi, A. (1987). Educare alla complessità del reale, in Scuola e città, 38.

Wallerstein, I. (2006). Comprendere il mondo. Introduzione all'analisi dei sistema-mondo. Trieste: Asterios editore.

Watzlawick, P., Beavin, J.H., Jackson D.D. (1967). Pragmatica della comunicazione umana. Roma: AstrolabioRoma.

Zizek, S. (2012). Benvenuti in tempi interessanti. Milano: Ponte delle Grazie. 